\title{
On a 'Mysterious' Case of a Quadratic Hamiltonian
}

\author{
Sergei SAKOVICH
}

Institute of Physics, National Academy of Sciences, 220072 Minsk, Belarus

E-mail:saks@tut.by

Received June 02, 2006, in final form July 18, 2006; Published online July 28, 2006

Original article is available at http://www.emis.de/journals/SIGMA/2006/Paper064/

\begin{abstract}
We show that one of the five cases of a quadratic Hamiltonian, which were recently selected by Sokolov and Wolf who used the Kovalevskaya-Lyapunov test, fails to pass the Painlevé test for integrability.
\end{abstract}

Key words: Hamiltonian system; nonintegrability; singularity analysis

2000 Mathematics Subject Classification: 70H07; 37J30; 34M35

\section{Introduction}

Recently, Sokolov and Wolf [1] applied the Kovalevskaya-Lyapunov test for integrability to a class of quadratic Hamiltonians and selected in this way five cases, out of which three cases were previously known to be integrable and one case turned out to be a new integrable Hamiltonian on $s o(3,1)$ with an additional sixth-degree polynomial integral. Integrability of the fifth case remained unknown, and the authors of [1] wrote the following:

Case (e) is a mysterious one. We have verified that the Hamiltonian has no additional polynomial integrals of degrees less than or equal to 8 . On the other hand, on all Kowalewski solutions all Kowalewski exponents are integers. It would be interesting to verify whether the equations of motion in case (e) satisfy the standard Painlevé test.

In the present short note, we apply the Painlevé test for integrability to this 'mysterious' case (e) and show that it must be nonintegrable due to some movable logarithmic branching of solutions. We use the Ablowitz-Ramani-Segur algorithm of singularity analysis of ODEs [2] (see also the review [3]).

\section{The studied quadratic Hamiltonian}

Sokolov and Wolf [1] considered the following family of Poisson brackets:

$$
\left\{M_{i}, M_{j}\right\}=\varepsilon_{i j k} M_{k}, \quad\left\{M_{i}, \gamma_{j}\right\}=\varepsilon_{i j k} \gamma_{k}, \quad\left\{\gamma_{i}, \gamma_{j}\right\}=\kappa \varepsilon_{i j k} M_{k},
$$

where $M_{i}$ and $\gamma_{i}$ are components of three-dimensional vectors $\boldsymbol{M}$ and $\boldsymbol{\Gamma}, \varepsilon_{i j k}$ is the totally skew-symmetric tensor, and $\kappa$ is a parameter. The cases $\kappa>0$ and $\kappa<0$ correspond to the $s o(4)$ and $s o(3,1)$ Lie algebras, whereas the $e(3)$ Lie algebra case $\kappa=0$ was not studied in [1]. Since the brackets (1) have two Casimir functions, $J_{1}=(\boldsymbol{M}, \boldsymbol{\Gamma})$ and $J_{2}=\kappa|\boldsymbol{M}|^{2}+$

$|\boldsymbol{\Gamma}|^{2}$ (with standard notations for the vector dot product and module), only one additional integral functionally independent of the Hamiltonian and the Casimir functions is required for the Liouville integrability of the equations of motion. 
The special class of Hamiltonians studied by Sokolov and Wolf [1] has the following form:

$$
H=c_{1}(\boldsymbol{a}, \boldsymbol{b})|\boldsymbol{M}|^{2}+c_{2}(\boldsymbol{a}, \boldsymbol{M})(\boldsymbol{b}, \boldsymbol{M})+(\boldsymbol{b}, \boldsymbol{M} \times \boldsymbol{\Gamma}),
$$

where $c_{1}$ and $c_{2}$ are parameters, the constant vectors $\boldsymbol{a}$ and $\boldsymbol{b}$ are taken to be $\boldsymbol{a}=\left(a_{1}, 0, a_{3}\right)$ and $\boldsymbol{b}=(0,0,1), a_{1}^{2}+a_{3}^{2}=-\kappa$, and $\times$ denotes the vector skew product. The Hamiltonians (2), referred to as 'vectorial' Hamiltonians in [1], belong to a wider class of quadratic Hamiltonians which have numerous applications (two-spin interactions, motion of a three-dimensional rigid body in a constant-curvature space or in an ideal fluid, motion of a body with ellipsoidal cavities filled with an ideal fluid, etc).

Sokolov and Wolf [1] applied the Kovalevskaya-Lyapunov test for integrability to the class of Hamiltonians (2) and selected in this way the following five cases: (a) $c_{1}$ is arbitrary, $c_{2}=0$; (b) $c_{1}=1, c_{2}=-2$; (c) $c_{1}=1, c_{2}=-1$; (d) $c_{1}=1, c_{2}=-\frac{1}{2}$; (e) $c_{1}=1, c_{2}=1$. It was pointed out in [1] that the case (a) obviously possesses the linear additional integral $I=(\boldsymbol{b}, \boldsymbol{M})$, whereas the cases (b) and (c) correspond to two recently discovered integrable Hamiltonian systems with polynomial additional integrals of degrees three [4] and four [5], respectively. It is remarkable that the case (d) turned out to be a new integrable quadratic Hamiltonian discovered by the Kovalevskaya-Lyapunov test, with an additional sixth-degree polynomial integral [1]. However, in the case (e), which also passed the Kovalevskaya-Lyapunov test well, Sokolov and Wolf [1] failed to find an additional integral.

In the next section, we will show that the equations of motion in this 'mysterious' case (e) fail to pass the Painlevé test and must be nonintegrable in the Liouville sense. It is worthwhile to remember, however, that the fact of not passing the Painlevé test does not necessarily imply nonintegrability of a tested system, and the way is essential how the test is failed. If the Painlevé test is failed at its first step, when the leading exponents are determined, or at its second step, when the positions of resonances are determined, it is sometimes possible to improve the behavior of solutions by a transformation of variables so that the transformed system passes the Painlevé test well. In particular, rational leading exponents and rational positions of resonances are allowed by the so-called weak Painlevé property which may [6] - as well as may not [7] correspond to integrability. However, if the Painlevé test is failed at its third step, when the compatibility of recursion relations is checked at the resonances, one only have to introduce some logarithmic terms into the expansions of solutions, and this logarithmic branching of solutions is generally believed to be a clear symptom of nonintegrability [8]. We will see that, in the case (e) of the Hamiltonian (2), the Painlevé test is failed in this last - hopeless - way.

\section{Demystifying the 'mysterious' case}

We are going to show that the system of six ODEs

$$
\begin{aligned}
& \dot{m}_{1}=m_{1} m_{2}+2 a m_{2} m_{3}-m_{1} g_{3}+m_{3} g_{1}, \\
& \dot{m}_{2}=-m_{1}^{2}-2 a m_{1} m_{3}+m_{3}^{2}-m_{2} g_{3}+m_{3} g_{2}, \\
& \dot{m}_{3}=-m_{2} m_{3}, \\
& \dot{g}_{1}=\left(1+a^{2}\right) m_{1} m_{3}+m_{1} g_{2}-2 a m_{2} g_{3}+4 a m_{3} g_{2}+g_{1} g_{3}, \\
& \dot{g}_{2}=\left(1+a^{2}\right) m_{2} m_{3}-m_{1} g_{1}+2 a m_{1} g_{3}-4 a m_{3} g_{1}+m_{3} g_{3}+g_{2} g_{3}, \\
& \dot{g}_{3}=-\left(1+a^{2}\right)\left(m_{1}^{2}+m_{2}^{2}\right)-2 a m_{1} g_{2}+2 a m_{2} g_{1}-m_{3} g_{2}-g_{1}^{2}-g_{2}^{2},
\end{aligned}
$$

where the dot denotes $\frac{d}{d t}$ and $a$ is a parameter, must be nonintegrable unless $a=0$. The system (3) represents the equations of motion in the case (e) of the Hamiltonian (2). The notations $m_{i}, g_{i}$ and $a$ used in (3) correspond to $M_{i}, \gamma_{i}$ and $a_{3}$ used in (2), respectively, whereas 
for the nonzero parameter $a_{1}$ of (2) we have set $a_{1}=1$ by rescaling $M_{i}$ and $\gamma_{i}$ (note that the case (e) with $a_{1}=0$ falls under the case (a) which possesses a linear additional integral).

Assuming that $a \neq 0$ in (3), where all quantities are considered as complex-valued from now on, and using the expansions

$$
\begin{aligned}
& m_{i}=m_{i, 0} \phi^{\alpha_{i}}+\cdots+m_{i, r} \phi^{r+\alpha_{i}}+\cdots, \\
& g_{i}=g_{i, 0} \phi^{\beta_{i}}+\cdots+g_{i, r} \phi^{r+\beta_{i}}+\cdots, \\
& i=1,2,3, \quad \phi=t-t_{0},
\end{aligned}
$$

where $m_{i, j}, g_{i, j}, \alpha_{i}, \beta_{i}$ and $t_{0}$ are constants, we find the following singular branches and positions $r$ of resonances in them (note that we do not consider the possibility of $a^{2}=-1$, because it corresponds to the case of $\kappa=0$, i.e. the $e(3)$ Lie algebra, which was not studied in [1]):

$$
\begin{aligned}
& \alpha_{i}=\beta_{i}=-1, \quad i=1,2,3, \\
& m_{1,0}=\frac{ \pm a}{\sqrt{-1-a^{2}}}, \quad m_{2,0}=1, \quad m_{3,0}=\frac{\mp 1}{\sqrt{-1-a^{2}}}, \\
& g_{1,0}=-a, \quad g_{2,0}=\mp \sqrt{-1-a^{2}}, \quad g_{3,0}=1, \\
& r=-1,0,1,2,2,2 ;
\end{aligned}
$$

and

$$
\begin{aligned}
& \alpha_{i}=\beta_{i}=-1, \quad i=1,2,3, \\
& m_{1,0}=\frac{ \pm a}{\sqrt{-1-a^{2}}}, \quad m_{2,0}=1, \quad m_{3,0}=\frac{\mp 1}{\sqrt{-1-a^{2}}}, \\
& g_{1,0}=a, \quad g_{2,0}= \pm \sqrt{-1-a^{2}}, \quad g_{3,0}=-1, \\
& r=-2,-1,1,2,2,4 .
\end{aligned}
$$

Let us look at the branch (5) first. According to the positions of resonances, this branch must be a generic one. We see, however, that the position of one resonance is $r=0$ there, whereas all the coefficients $m_{1,0}, m_{2,0}, m_{3,0}, g_{1,0}, g_{2,0}$ and $g_{3,0}$ turn out to be fixed. This means that, in the case of (5), the recursion relations for the coefficients of the expansions (4) have a nontrivial compatibility condition right in the position $r=0$, and we have to modify (4) by introducing additional logarithmic terms, starting from the terms proportional to $\phi^{-1} \log \phi$. Suppose we do not do this and think that the branch (5) represents not the general solution but a class of special solutions. Then we find that no compatibility condition appears at the resonance $r=1$, where the coefficient $m_{1,1}$ remains arbitrary. However, at the triple resonance $r=2$, where the coefficients $m_{1,2}, m_{2,2}$ and $g_{1,2}$ remain arbitrary, the nontrivial compatibility condition $m_{1,1}=0$ appears, and we are again forced to introduce logarithmic terms into the expansions of solutions.

The branch (6) gives us the same information: solutions of the system (3) with $a \neq 0$ possess movable logarithmic singularities. In this branch, the nontrivial compatibility condition

$$
\begin{aligned}
m_{1,1}^{2}\left[2 a \sqrt{-1-a^{2}}\left(2+a^{2}\right)^{2} m_{1,2}\right. \\
\left.\quad+\left(2+a^{2}\right)^{2}\left(3+2 a^{2}\right) m_{2,2}+\left(4+9 a^{2}+5 a^{4}\right) m_{1,1}^{2}\right]=0
\end{aligned}
$$

appears at the resonance $r=4$.

Consequently, the system (3) with $a \neq 0$ fails to pass the Painlevé test for integrability, and this, in its turn, explains why Sokolov and Wolf [1] failed to find an additional integral for the case (e) $c_{1}=1, c_{2}=1$ of the quadratic Hamiltonian (2).

The case of (3) with $a=0$ is different: it passes the Painlevé test well, as one can easily verify. However, the fact of integrability of the system (3) with $a=0$ is not new: the case (e) with $a_{3}=0$ of the Hamiltonian (2) falls under the case (c) with a fourth-degree additional polynomial integral (we are indebted to Prof. T. Wolf who kindly explained this point to us). 


\section{Conclusion}

We have shown that the 'mysterious' case (e) $c_{1}=1, c_{2}=1$ of the quadratic Hamiltonian (2), selected by Sokolov and Wolf [1] who used the Kovalevskaya-Lyapunov test, fails to pass the Painlevé test for integrability. It is worthwhile to remember that, from the standpoint of the Painlevé test [2,3], the Kovalevskaya-Lyapunov test only examines the positions of resonances for a studied system and is sensitive to the nondominant algebraic branching of solutions, whereas the Painlevé test also verifies the compatibility of recursion relations at the resonances and can detect the nondominant logarithmic branching of solutions which is the strongest indication of nonintegrability. We can add that it happens quite frequently in the singularity analysis practice that the positions of resonances are good but the recursion relations are incompatible. For example, in the integrability study of symmetrically coupled higher-order nonlinear Schrödinger equations [9], 23 distinct cases with integer positions of all resonances were found, but the recursion relations turned out to be compatible in only one case out of those 23 cases (see the table in [10] for more details).

[1] Sokolov V.V., Wolf T., Integrable quadratic classical Hamiltonians on so(4) and so(3,1), J. Phys. A: Math. Gen., 2006, V.39, 1915-1926, nlin.SI/0405066.

[2] Ablowitz M.J., Ramani A., Segur H., A connection between nonlinear evolution equations and ordinary differential equations of P-type. I, J. Math. Phys., 1980, V.21, 715-721.

[3] Ramani A., Grammaticos B., Bountis T., The Painlevé property and singularity analysis of integrable and non-integrable systems, Phys. Rep., 1989, V.180, 159-245.

[4] Tsiganov A.V., Goremykin O.V., Integrable systems on $s o(4)$ related with $X X X$ spin chains with boundaries, J. Phys. A: Math. Gen., 2004, V.37, 4843-4849, nlin.SI/0310049.

[5] Sokolov V.V., On a class of quadratic Hamiltonians on so(4), Dokl. Akad. Nauk, 2004, V.394, $602-605$ (in Russian).

[6] Ramani A., Dorizzi B., Grammaticos B., Painlevé conjecture revisited, Phys. Rev. Lett., 1982, V.49, 15391541.

[7] Grammaticos B., Dorizzi B., Ramani A., Integrability of Hamiltonians with third- and fourth-degree polynomial potentials, J. Math. Phys., 1983, V.24, 2289-2295.

[8] Ablowitz M.J., Clarkson P.A., Solitons, nonlinear evolution equations and inverse scattering, Cambridge, Cambridge University Press, 1991.

[9] Sakovich S.Yu., Tsuchida T., Symmetrically coupled higher-order nonlinear Schrödinger equations: singularity analysis and integrability, J. Phys. A: Math. Gen., 2000, V.33, 7217-7226, nlin.SI/0006004.

[10] Sakovich S.Yu., Tsuchida T., Coupled higher-order nonlinear Schrödinger equations: a new integrable case via the singularity analysis, nlin.SI/0002023. 